\title{
Maternal depression in rural Pakistan: the protective associations with cultural postpartum practices
}

\author{
Katherine LeMasters ${ }^{1,2^{*}}$ (D), Nafeesa Andrabi ${ }^{2,3}$, Lauren Zalla', Ashley Hagaman", Esther O. Chung ${ }^{1,2}$, John A. Gallis ${ }^{5,6}$,
} Elizabeth L. Turner ${ }^{5,6}$, Sonia Bhalotra ${ }^{7}$, Siham Sikander ${ }^{8,9}$ and Joanna Maselko ${ }^{1,2}$

\begin{abstract}
Background: Traditional postpartum practices are intended to provide care to mothers, but there is mixed evidence concerning their impact on postpartum depression (PPD). It remains unknown if there is a unique impact of postpartum practices on PPD separately from other types of social support, or if practices differentially affect those with existing prenatal depression. In Pakistan, chilla ( $\Delta \mathrm{JZ}_{\mathrm{Z}}$ ) is a traditional postpartum practice in which women receive relief from household work, additional familial support, and supplemental food for up to 40 days postpartum. This study aims to understand if chilla protects against PPD independent of other support and whether this relationship varies by prenatal depression status.
\end{abstract}

Methods: Data come from the Bachpan cohort study in rural Pakistan. Chilla participation and social support (Multidimensional Scale of Perceived Social Support) were assessed at 3 months postpartum. Women were assessed for major depressive episodes (MDE) with the Structured Clinical Interview, DSM-IV and for depression symptom severity with the Patient Health Questionnaire (PHQ-9) in their third trimester and at 6 months postpartum. Adjusted linear mixed models were used to assess the relationship between chilla participation and PPD.

Results: Eighty-nine percent of women $(N=786)$ participated in chilla and almost $70 \%$ of those that participated took part in all of chilla's aspects. In adjusted models, chilla participation was inversely related to MDE (OR $=0.56$; $95 \% \mathrm{Cl}=0.31,1.03$ ) and symptom severity (Mean Difference $(\mathrm{MD})=-1.54 ; 95 \% \mathrm{Cl}$ : $-2.94,-0.14$ ). Chilla participation was associated with lower odds of MDE $(O R=0.44 ; 95 \% \mathrm{Cl}=0.20,0.97)$ among those not prenatally depressed and with lower symptom severity among those prenatally depressed ( $M D=-2.05 ; 95 \% \mathrm{Cl}:-3.81,-0.49)$.

Conclusions: Chilla is inversely associated with both MDE and symptom severity at 6 months postpartum above and beyond social support. Specifically, chilla is inversely associated with MDE among those not prenatally depressed and with lower symptom severity among those prenatally depressed. This relationship signals an opportunity for interventions aimed at preventing and treating PPD in this region to draw upon chilla and similar traditional postpartum practices in creating community-based, low-cost, sustainable interventions for maternal mental health.

Trial registration: NCT02111915. Registered 18 September 2015. NCT02658994. Registered 22 January 2016. Both trials were prospectively registered.

Keywords: Postpartum, Perinatal, Depression, Mental health, Practices, Support, Pakistan

\footnotetext{
* Correspondence: Katherine.lemasters@unc.edu

'Department of Epidemiology, Gillings School of Global Public Health,

University of North Carolina at Chapel Hill, McGavran-Greenberg Hall, CB\#

7435, Chapel Hill, North Carolina NC 27599, USA

${ }^{2}$ Carolina Population Center, University of North Carolina at Chapel Hill, 123

W. Franklin St, Chapel Hill, North Carolina NC 27516, USA

Full list of author information is available at the end of the article
}

(c) The Author(s). 2020 Open Access This article is distributed under the terms of the Creative Commons Attribution 4.0 International License (http://creativecommons.org/licenses/by/4.0/), which permits unrestricted use, distribution, and reproduction in any medium, provided you give appropriate credit to the original author(s) and the source, provide a link to the Creative Commons license, and indicate if changes were made. The Creative Commons Public Domain Dedication waiver (http://creativecommons.org/publicdomain/zero/1.0/) applies to the data made available in this article, unless otherwise stated. 


\section{Background}

The postpartum period is one of vulnerability for women's mental health, which may be protected through preexisting sociocultural practices. The importance of traditional postpartum practices to women's postpartum experience has long been documented throughout regions of the world [1]. While such practices vary cross-culturally, the first months after giving birth are widely acknowledged as distinct from normal life and as a recuperative time [2]. Postpartum practices are richly documented in Asian, Hispanic, African, and Jewish cultures and are described by anthropologists and cultural psychiatrists as customs that "mother the mother" through formalized social support [1, 3-5]. They are intended to provide care to mothers by limiting their household activities, providing a special diet, taking care of their needs, and easing their transition into motherhood [1].

Such practices may also have long term benefits on maternal and infant health, including maternal mental health, and need to be considered when thinking about protections against postpartum depression (PPD) $[1,4,6]$. PPD is defined as depression beginning in or extending into the postpartum period, up to 1 year after giving birth, and can impair women's functioning and the development of her children [7]. In low- and middle-income countries (LMIC), the overall pooled prevalence of PPD is between $18.7-19.8 \%[8,9]$. Though postpartum practices in Asian cultures are intended as supportive mechanisms for new mothers and the baby, there is mixed evidence concerning the impact of postpartum practices on PPD.

For instance, a systematic review on postpartum practices found that appropriate and wanted support may be protective against PPD. [10] In Taiwan, research suggests that women who engaged in traditional practices were less likely to exhibit physical symptoms and suffer from PPD at four to 6 weeks postpartum [11]. Yet, another systematic review of 16 studies reporting on traditional practices and PPD in Asian cultures found mixed results with eight studies reporting protective associations, four reporting negative associations, and the remaining studies were inconclusive [12]. Additional studies from Japan and Vietnam reported null associations and another from Singapore suggests that postpartum practices were correlated with higher depression levels [13-15].

It is likely that the potential effects of postpartum practices on PPD are closely tied to the social support components of such practices [10]. Social support refers to any type of assistance received from others, and is frequently categorized into instrumental and emotional support. There are known behavioral, psychological, and biological mechanisms through which both instrumental and emotional support act to influence health outcomes [16]. Postpartum practices provide instrumental support, such as a supplemented diet and relief from chores, and emotional support, such as a bolstered sense of self-worth. These social support components of postpartum practices may be driving the association between participation and reduced risk of PPD.

Prior literature has found that social support is often considered the most important aspect of participation in postpartum practices [10]. Participation may be less beneficial when support is less positive, such as when the practice creates conflict with a mother-in-law, or when support is unwanted. Yet, few studies that assess participation in postpartum practices have measured social support separately to tease out the complex relationships between engagement in postpartum practices and social support.

Additionally, prior work has focused on the role that postpartum practices play in preventing PPD onset, but has not assessed how these practices reduce pre-existing depression symptom severity $[10,12]$. The pooled prevalence of women with PPD in LMIC is between 18.7-19.8\% and the pooled prevalence of women diagnosed prenatally is between 15.6$19.2 \%$, indicating that many women enter the postpartum period and begin their postpartum practices already experiencing depression $[8,9]$. Thus, there is a need to understand if postpartum practices reduce depressive symptom severity among those prenatally depressed as well as prevent the onset of new symptoms.

In Pakistan, postpartum practices are referred to as chilla ( $ه$ (z:) and are common after a women's first birth [17]. Chilla is defined as a 40-day period of confinement after childbirth in which a woman returns to her mother's home, is fed fortifying foods, is exempt from household responsibilities, stays indoors, and receives additional support. Yet, not all women return to their maternal home during this period; many resume chores within 7 days postpartum, and women may only receive unsolicited social support [18]. Additionally, PPD is estimated to be $30.9 \%$ in Pakistan, notably higher than the estimated prevalence of $18.7-19.8 \%$ in LMIC [19]. To date, one study in Pakistan has found chilla to be protective against PPD. [20] Given the dearth of evidence and inconclusiveness, our study of mothers in rural Pakistan aims to understand [1] how chilla affects depression diagnosis and depressive symptom severity, [2] whether this relationship exists above and beyond perceived social support and [3] whether this relationship varies by prenatal depression status.

\section{Methods}

\section{Study design and participants}

Data come from the Bachpan study, a cluster randomized perinatal depression trial nested in a longitudinal birth cohort in rural Pakistan. The study is representative of a typical low-socioeconomic rural area in the north of the Punjab Province. A more detailed description of the study is available elsewhere [21]. Briefly, pregnant women in 40 village clusters were identified and invited to be screened for depression using the Patient Health Questionnaire (PHQ-9). 
Women who scored 10 or higher were invited to participate in the study; roughly one in three women who scored less than 10 were asked to participate in the study [21]. Given unequal probabilities of selection into the study, sampling weights were used to represent the population of pregnant women in the area [21]. Women were then followed up at three, six, 12, 24, and 36 months postpartum through inperson interviews. We used data from pregnancy (baseline), three, and 6 months postpartum. Of the 1154 women interviewed at baseline, 331 were not interviewed at the threemonth follow-up visit, resulting in 823 women in our analytic sample.

\section{Measures}

\section{Outcomes}

Our main outcomes of interest were major depressive episode (MDE) and depressive symptom severity at 6 months. We utilized the Structured Clinical Interview for the Diagnostic and Statistical Manual of Mental Disorders' (SCID-IV, hereafter referred to as SCID) Module for Current Major Depressive Episode to evaluate MDE [22]. The major depressive section of the SCID has been translated into Urdu, culturally adapted for Pakistan, and has been used cross-culturally with pregnant women $[23,24]$. The SCID was collected at baseline, three, and 6 months. The PHQ- 9 screens for depressive symptoms using nine items with a score ranging from zero to three for each item; individuals can receive a maximum score of 27. A 10-point cutoff is commonly used to indicate symptoms reaching a clinically significant level [25]. This measure has demonstrated acceptable criterion-related validity and reliability for screening depressive symptoms in our population of community-based Pakistani pregnant women [25]. The PHQ-9 was collected at baseline, three, and 6 months and treated as a continuous variable.

\section{Exposure}

At the 3 month postpartum interview, women were asked whether they participated in chilla. If yes, they were asked a series of questions about their chilla experience, which were based on a prior Pakistani study [26]. These included [1] the duration of their chilla (approximately one-week intervals from 1 to 40 days); three questions about chilla components: [2] being relieved from chores, [3] receiving additional female support, and [4] receiving a supplemented diet; and [5] a final question about whether the woman was satisfied with her chilla. Our primary analyses used the binary variable for chilla participation, as there was little variation in the component indicators [20]. Additional sensitivity analyses were conducted to assess whether specific components of chilla participation were associated with MDE at 6 months.

\section{Covariates}

The Multidimensional Scale of Perceived Social Support (MSPSS) is used globally and captures perceived support from family, friends, and significant others [27]. Informed by a prior validation study of the Urdu translation of the MSPSS among new mothers in rural Pakistan, we summed the scores of all 12 items to obtain a single summary measure of perceived social support [28]. The MSPSS was measured at 3 months postpartum.

We also adjusted for potential baseline confounders a priori: maternal age (years), maternal education (years), parity, socioeconomic status (SES) (a composite of household assets), and living with mother-in-law [29]. Our analysis also controlled for baseline outcome measures (e.g. SCID at baseline was included when SCID at 6 months was the outcome). In our models, we adjusted for study arm and the number of people living in a room, as this was associated with missingness at three- and six-month interviews.

\section{Statistical analysis}

We first present descriptive statistics for the sample characteristics and mean differences in these characteristics by chilla participation. Our primary analyses assessed the relationship between chilla participation and MDE (SCID) and depressive symptom severity (PHQ-9) at 6 months separately. We utilized multilevel mixed effects models with a logit link for SCID and an identity link for PHQ-9. All models used a random intercept to account for clustering at the village level. Given the unequal probabilities of selection into the study, sampling weights were used in models and descriptive statistics to represent the underlying population of pregnant women in the area. Our analyses were stratified by MDE at baseline, given that the relationship of chilla on sixmonth depression may be different among depressed and non-depressed mothers. We conducted two sensitivity analyses: [1] to examine the associations between specific components of chilla and MDE (SCID) and [2] to assess whether inclusion of MSPSS attenuated the association between chilla and subsequent depression. All analyses were conducted using Stata 15.

\section{Results \\ Descriptive statistics}

Our final analytic sample included 823 women who had complete data for baseline, three, and six-month timepoints. A comparison of baseline characteristics among women with and without available data is Table 4 in Appendix. After applying population-representative weights, women were, on average, 26.62 years old and over half of women (51.38\%) had at least a secondary education (Table 1). Most had at least one other child $(61.81 \%)$ and lived with their motherin-law (68.56\%). Mean social support (MSPSS) score at 3 months was 4.74 (SD: 1.15). Thirty-seven percent of women were diagnosed with MDE (SCID) at baseline and 23.06\% were diagnosed at 6 months postpartum. Mean PHQ-9 score was 8.73 at baseline (SD: 6.68$)$ and 4.70 at 6 months (SD: 5.44). 
Table 1 Demographic, chilla, and depression characteristics, Bachpan cohort, Pakistan ${ }^{a}$

\begin{tabular}{|c|c|c|c|c|}
\hline & Mean or $\mathrm{N}$ & SD or $\%$ & Min & Max \\
\hline \multicolumn{5}{|c|}{ Demographic Characteristics $(N=823)$} \\
\hline Maternal Age & 26.62 & 4.43 & 18 & 40 \\
\hline \multicolumn{5}{|l|}{ Maternal Education } \\
\hline None & 111 & 12.23 & & \\
\hline Primary $(1-5)$ & 159 & 17.46 & & \\
\hline Middle (6-8) & 157 & 18.94 & & \\
\hline Secondary $(9,10)$ & 209 & 25.96 & & \\
\hline Higher Secondary $(11,12)$ & 84 & 10.92 & & \\
\hline Tertiary (> 12) & 103 & 14.50 & & \\
\hline \multicolumn{5}{|l|}{ Parity } \\
\hline First Pregnancy & 238 & 30.60 & & \\
\hline One or more children & 585 & 69.40 & & \\
\hline \multicolumn{5}{|l|}{ SES Quintiles } \\
\hline Lowest & 153 & 15.99 & & \\
\hline Lower Middle & 163 & 18.56 & & \\
\hline Middle & 171 & 21.13 & & \\
\hline Upper Middle & 165 & 21.64 & & \\
\hline Highest & 171 & 22.68 & & \\
\hline \multicolumn{5}{|l|}{ Mother-in-law } \\
\hline Yes & 557 & 68.56 & & \\
\hline People in Room & 2.37 & 1.73 & 0 & 23 \\
\hline MSPSS 3 month & 4.74 & 1.15 & 1 & 7 \\
\hline \multicolumn{5}{|l|}{ Chilla Characteristics $(N=729)$} \\
\hline \multicolumn{5}{|l|}{ Chilla } \\
\hline Yes & 729 & 89.44 & & \\
\hline \multicolumn{5}{|l|}{ Duration } \\
\hline $1-7$ & 71 & 9.06 & & \\
\hline $8-14$ & 78 & 9.58 & & \\
\hline $15-20$ & 120 & 15.79 & & \\
\hline $21-25$ & 38 & 4.89 & & \\
\hline $26-30$ & 53 & 7.01 & & \\
\hline $31-35$ & 30 & 4.25 & & \\
\hline $36-40$ & 339 & 49.42 & & \\
\hline \multicolumn{5}{|l|}{ Chores } \\
\hline Yes & 620 & 85.30 & & \\
\hline \multicolumn{5}{|l|}{ Female Support } \\
\hline Yes & 686 & 94.66 & & \\
\hline \multicolumn{5}{|l|}{ Diet } \\
\hline Yes & 679 & 93.84 & & \\
\hline \multicolumn{5}{|l|}{ Satisfaction } \\
\hline Yes & 602 & 84.79 & & \\
\hline \multicolumn{5}{|c|}{ Depression Characteristics $(N=823)$} \\
\hline SCID (Baseline) & 405 & 37.54 & & \\
\hline
\end{tabular}

Table 1 Demographic, chilla, and depression characteristics, Bachpan cohort, Pakistan ${ }^{\text {a }}$ (Continued)

\begin{tabular}{cllll}
\hline & Mean or N & SD or \% & Min & Max \\
\hline SCID (Six months) & 217 & 23.06 & & \\
PHQ-9 (Baseline) & 8.73 & 6.68 & 0 & 27 \\
PHQ-9 (6 months) & 4.70 & 5.44 & 0 & 27 \\
\hline
\end{tabular}

a Percentages are based on weighted data to account for the sampling design in which 1 in every 3 non-depressed pregnant women were eligible for study inclusion; Abbreviations: Multidimensional Scale of Perceived Social Support (MSPSS), Socioeconomic Status (SES), Patient Health Questionnaire (PHQ-9), Structured Clinical Interview for DSM-IV (SCID)

\section{Chilla participation}

The majority of women $(89.44 \%)$ participated in chilla and over $80 \%$ were satisfied with their chilla experience (Table 1 ). Most participated for at least 26 days (60.68\%) and almost $70 \%$ participated in all three components (relief from chores, additional female support, supplemented diet). Women who participated in chilla had, on average, more education, higher SES, were more likely to be in their first pregnancy, and more baseline social support. They also had fewer MDEs at baseline and lower depression symptom severity (Table 2). We also assessed whether baseline MDE (SCID) predicted chilla participation and chilla components. More women who were non-depressed at baseline participated in chilla, had longer chilla duration, and were more satisfied with their chilla experience (Table 5 in Appendix).

\section{Symptom severity and MDE diagnosis}

In unadjusted models, chilla participation was inversely related to both MDE (Table 3 - Panel A, Model 1: OR = 0.40; $95 \% \mathrm{CI}=0.23,0.71$ ) and symptom severity (Table 3 - Panel B, Model 1: Mean Difference (MD) $=-2.51$; 95\% CI: -4.20 ,0.82). After adjusting for baseline covariates, we found that these relationships hold for both MDE (Table 3 - Panel A, Model 2: $\mathrm{OR}=0.53$; $95 \% \mathrm{CI}=0.33,0.86)$ and symptom severity (Table 3 - Panel B, Model 2: MD = -2.03; 95\% CI: - 3.10,0.96). We then controlled for social support and, although our estimate for chilla becomes slightly less precise, chilla remains independently related to MDE (Table 3 - Panel A, Model 3: $\mathrm{OR}=0.56 ; 95 \% \mathrm{CI}=0.31,1.03$ ) and symptom severity (Table 3 - Panel B, Model 3: MD = -1.54; 95\% CI: - 2.94,0.14). We conducted sensitivity analyses to disentangle potentially different associations of chilla components and PPD, adjusting for other components, and found no significant differences among the components (Table 6 in Appendix); however, this is likely because the majority of women who participated in chilla participated in all three components.

To examine whether the association between chilla and depression differs by baseline MDE, we stratified models by baseline MDE diagnosis. In multivariable models that also included social support, we found that the association between chilla and MDE at 6 months was driven by those not clinically depressed at baseline (Table 3 - Panel A, Model 
Table 2 Background Characteristics by Chilla Participation, Bachpan cohort, Pakistan*

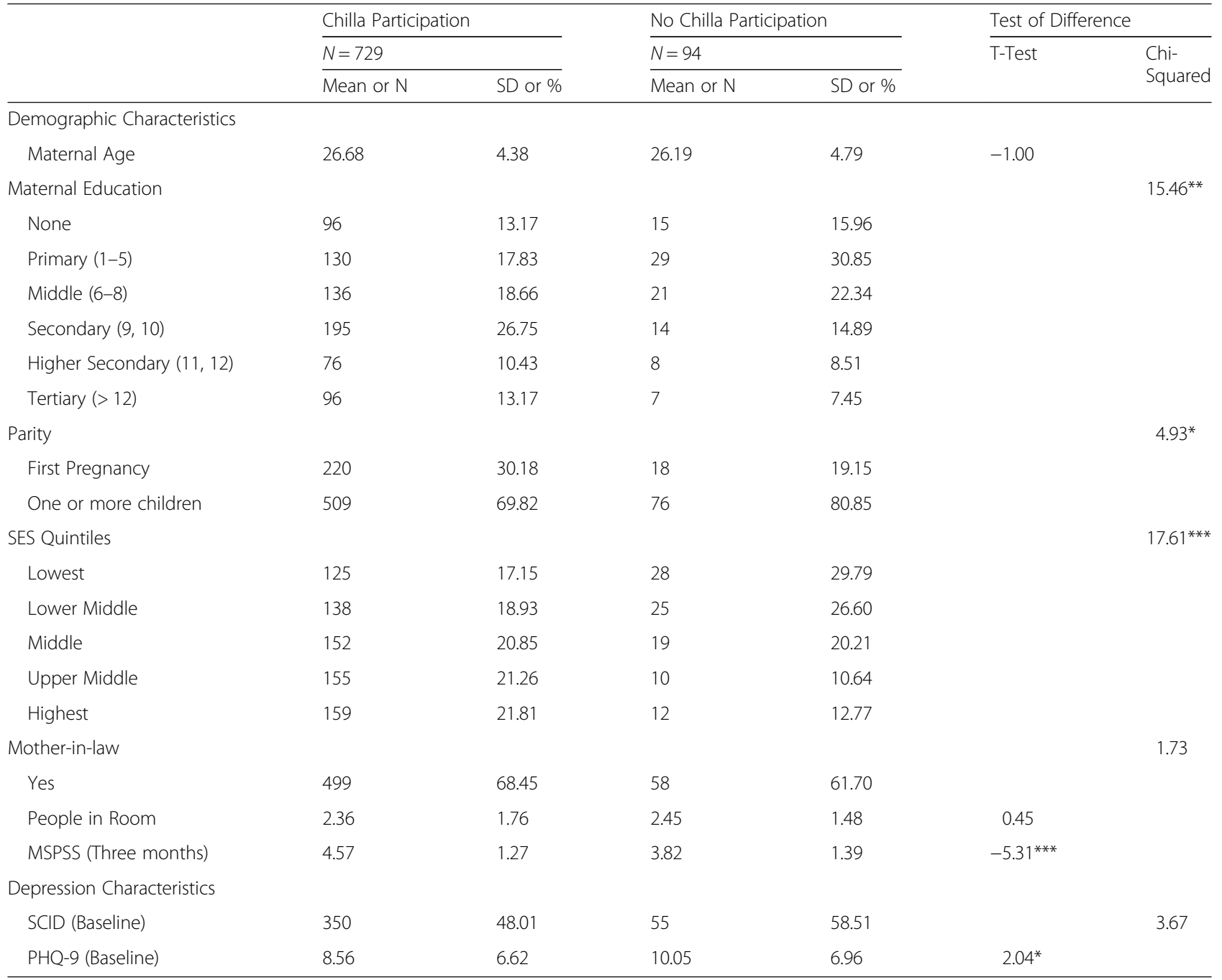

${ }^{*} p<.05,{ }^{* *}<.01,{ }^{* * *} p<.00$; Abbreviations: Multidimensional Scale of Perceived Social Support (MSPSS), Socioeconomic Status (SES); Patient Health Questionnaire $(P H Q-9)$, Structured Clinical Interview for DSM-IV (SCID)

D: $\mathrm{OR}=0.44 ; 95 \% \mathrm{CI}=0.20,0.97)$ as opposed to those who were clinically depressed at baseline (Table 3 - Panel A, Model E: $\mathrm{OR}=0.73 ; 95 \% \mathrm{CI}=0.39,1.34)$. Conversely, the association between chilla and symptom severity score was greater among those who had prenatal MDEs (Table 3 Panel B, Model E: MD = -2.05; 95\% CI: - 3.81,-0.49) compared to those who did not (Table 3 - Panel B, Model D: $\mathrm{MD}=-0.66 ; 95 \% \mathrm{CI}:-1.98,0.67)$.

\section{Discussion}

\section{Postpartum practices' differential effects}

Among women in rural Pakistan, we found that most participated in chilla and that most participated in all components for the majority of the traditional 40 days. Those with more education, first-time mothers, and those with higher SES were more likely to participate in chilla, as well as those without MDEs and those with lower depression symptom severity at baseline. Among chilla participants, those without baseline MDEs had more positive chilla experiences.

We found that postpartum practices hold promise for benefiting maternal mental health. Chilla was inversely associated with depression at 6 months postpartum above and beyond social support. We saw a larger effect on symptom severity (PHQ-9) among those prenatally diagnosed with depression, indicating that postpartum practices may be particularly beneficial for women with already-vulnerable mental health. We also saw a negative association between chilla participation and SCID diagnosis at 6 months among those without MDEs at baseline, meaning that chilla participation may be protective against the future development of depression. However, we did not see an association among those with prenatal MDEs. Thus, chilla participation may help prevent new onset of PPD, but may not be enough to lift women out of pre-existing depression. Our findings may point to why prior research has been inconclusive in 
Table 3 Chilla Participation and Postpartum Depression at Six Months, Bachpan cohort, Pakistan†

\begin{tabular}{|c|c|c|c|c|c|c|c|c|c|c|c|c|c|c|c|}
\hline \multirow{3}{*}{ Panel A: SCID+ } & \multirow{2}{*}{\multicolumn{3}{|c|}{$\begin{array}{l}\text { Model A: Full } \\
\text { Sample - Unadjusted } \\
N=823\end{array}$}} & \multirow{2}{*}{\multicolumn{3}{|c|}{$\begin{array}{l}\text { Model B: Full Sample - } \\
\text { MSPSS Excluded } \\
N=823\end{array}$}} & \multirow{2}{*}{\multicolumn{3}{|c|}{$\begin{array}{l}\text { Model C: Full } \\
\text { Sample - Adjusted } \\
N=823\end{array}$}} & \multirow{2}{*}{\multicolumn{3}{|c|}{$\begin{array}{l}\text { Model D: } \\
\text { Non-Depressed } \\
N=418\end{array}$}} & \multicolumn{3}{|c|}{ Model E: Depressed } \\
\hline & & & & & & & & & & & & & \multicolumn{3}{|l|}{$N=405$} \\
\hline & & & & & & & & & & & & & & & \\
\hline & OR & $\mathrm{Cl}$ & & OR & $\mathrm{Cl}$ & & OR & $\mathrm{Cl}$ & & OR & $\mathrm{Cl}$ & & OR & $\mathrm{Cl}$ & \\
\hline Chilla (yes) & 0.40 & 0.23 & 0.71 & $0.53^{*}$ & 0.33 & 0.86 & 0.56 & 0.31 & 1.03 & $0.44^{*}$ & 0.20 & 0.97 & 0.73 & 0.39 & 1.34 \\
\hline MSPSS & & & & & & & $0.72^{* * *}$ & 0.61 & 0.85 & $0.66^{* *}$ & 0.51 & 0.86 & $0.78^{*}$ & 0.65 & 0.93 \\
\hline SCID (Baseline) & & & & $2.47^{* * *}$ & 1.75 & 3.49 & $2.30^{* * *}$ & 1.62 & 3.26 & & & & & & \\
\hline Maternal Age & & & & 1.01 & 0.97 & 1.06 & 1.02 & 0.98 & 1.07 & 0.98 & 0.92 & 1.05 & 1.02 & 0.97 & 1.07 \\
\hline $\begin{array}{l}\text { Maternal } \\
\text { Education }\end{array}$ & & & & $0.83^{* *}$ & 0.72 & 0.94 & $0.81^{* *}$ & 0.70 & 0.94 & $0.81^{*}$ & 0.66 & 1.00 & 0.88 & 0.74 & 1.04 \\
\hline Parity & & & & 1.20 & 0.78 & 1.84 & 1.11 & 0.64 & 1.92 & 1.37 & 0.71 & 2.64 & 1.22 & 0.68 & 2.18 \\
\hline SES & & & & 0.88 & 0.76 & 1.02 & 0.91 & 0.75 & 1.11 & 1.01 & 0.79 & 1.28 & 0.84 & 0.70 & 1.01 \\
\hline Mother-in-law & & & & 1.15 & 0.79 & 1.69 & 1.26 & 0.84 & 1.88 & 1.46 & 0.76 & 2.80 & 1.08 & 0.66 & 1.76 \\
\hline People in Room & & & & 1.00 & 0.91 & 1.10 & 1.01 & 0.91 & 1.12 & 1.09 & 0.96 & 1.23 & 0.92 & 0.80 & 1.06 \\
\hline Study Arm & & & & 0.85 & 0.59 & 1.23 & 0.89 & 0.60 & 1.33 & 0.94 & 0.54 & 1.61 & 0.85 & 0.56 & 1.29 \\
\hline Intercept & 0.63 & 0.36 & 1.12 & 0.42 & 0.12 & 1.44 & 1.32 & 0.23 & 7.63 & 3.93 & 0.40 & 38.44 & 2.84 & 0.51 & 15.86 \\
\hline \multicolumn{16}{|l|}{ Panel B: PHQ-9++ } \\
\hline & Coefficient & $\mathrm{Cl}$ & & Coefficient & $\mathrm{Cl}$ & & Coefficient & $\mathrm{Cl}$ & & Coefficient & $\mathrm{Cl}$ & & Coefficient & $\mathrm{Cl}$ & \\
\hline Chilla (yes) & -2.51 & -4.20 & -0.82 & $-2.03^{* * *}$ & -3.10 & -0.96 & $-1.54^{*}$ & -2.94 & -0.14 & -0.66 & -1.98 & 0.67 & $-2.05^{*}$ & -3.81 & -0.49 \\
\hline MSPSS & & & & & & & $-0.85^{* * *}$ & -1.16 & $\begin{array}{l}- \\
0.55\end{array}$ & $-0.65^{* *}$ & $\begin{array}{l}- \\
1.06\end{array}$ & $\begin{array}{l}- \\
0.24\end{array}$ & $-0.93^{* * *}$ & $\begin{array}{l}- \\
1.38\end{array}$ & $\begin{array}{l}- \\
0.46\end{array}$ \\
\hline PHQ-9 (Baseline) & & & & $0.28^{* * *}$ & 0.22 & 0.33 & $0.25^{* * *}$ & 0.19 & 0.30 & $0.27^{* * *}$ & 0.17 & 0.36 & $0.21^{* * *}$ & 0.10 & 0.33 \\
\hline Maternal Age & & & & 0.04 & -0.04 & 0.13 & 0.04 & $\overline{-} .04$ & 0.12 & 0.02 & -0.08 & 0.12 & 0.04 & -0.09 & 0.19 \\
\hline $\begin{array}{l}\text { Maternal } \\
\text { Education }\end{array}$ & & & & -0.34 & -0.60 & $\begin{array}{l}- \\
0.07\end{array}$ & -0.22 & $\overline{0}-49$ & 0.05 & -0.23 & $\begin{array}{l}- \\
0.51\end{array}$ & 0.06 & -0.37 & $\begin{array}{l}- \\
0.81\end{array}$ & 0.07 \\
\hline Parity & & & & 0.09 & -0.76 & 0.93 & 0.12 & -0.55 & 0.78 & 0.21 & -0.68 & 1.10 & 0.20 & -1.34 & 1.00 \\
\hline SES & & & & -0.11 & -0.40 & 0.19 & 0.01 & -0.36 & 0.37 & 0.11 & -0.23 & 0.45 & -0.12 & -0.60 & 0.35 \\
\hline Mother-in-law & & & & 0.24 & -0.55 & 1.02 & 0.26 & -0.46 & 0.98 & 0.03 & -0.85 & 0.91 & 0.36 & -0.56 & 3.53 \\
\hline People in Room & & & & $0.04^{* * *}$ & -0.16 & 0.25 & 0.05 & -0.13 & 0.23 & -0.05 & -0.27 & 0.17 & 0.11 & -0.22 & 0.48 \\
\hline sStudy Arm & & & & -0.74 & -1.44 & -0.04 & -0.70 & -1.37 & -0.04 & -0.76 & $\begin{array}{l}- \\
1.52\end{array}$ & 0.00 & -0.75 & -1.88 & 0.31 \\
\hline Intercept & 6.40 & 4.71 & 8.08 & $4.02^{*}$ & 1.45 & 6.59 & 7.34 & 4.24 & 10.44 & 6.13 & 2.67 & 9.58 & 8.98 & 4.52 & 14.00 \\
\hline
\end{tabular}

† Models A-C used sampling weights and all models accounted for clustering due to the study design; ${ }^{*} p<.05,{ }^{* *}<.01,{ }^{* * *} p<.001 ;+$ Panel A used multilevel mixed-effects logistic regression with random effects to account for clustering; ++ Panel B used generalized linear mixed models with random effects to account for clustering; Abbreviations: Multidimensional Scale of Perceived Social Support (MSPSS), Socioeconomic Status (SES), Patient Health Questionnaire (PHQ-9), Structured Clinical Interview for DSM-IV (SCID)

understanding the relationship between postpartum practices and maternal depression, as such practices may have differential effects depending on both the depression measure used (i.e., MDE or symptom severity) and women's depressive history. Additionally, our findings may be specific to using the PHQ-9 and SCID. We used the PHQ-9 so that it would be generalizable across the prenatal, postnatal, and longer-term postpartum periods given the goal of the larger study. We chose to use the SCIDIV given its cross-cultural validation and use in this study setting [24, 30, 31]. Thus, studies using other instruments to assess symptom severity and MDE may generate different results.

\section{Chilla beyond social support}

The negative association between participation in chilla and PPD in our study sample is consistent with prior findings in Pakistan, which found that the relative risk of depression among mothers who participated in chilla compared to those who did not participate was 0.4 (95\% CI 0.3,0.6) [20]. Studies conducted in China, Vietnam, and Malaysia have also shown negative associations between participation in traditional postpartum practices and PPD. [14, 32, 33] Most often, social support is put forward as the mechanism linking traditional postpartum practices to reduced risk of PPD. For example, a study of mothers in Hong Kong found that the cultural practice of peiyue was associated with both better 
social support and lower risk of PPD. [32] After controlling for social support, peiyue was no longer associated with PPD, suggesting that social support is the "active ingredient" of peiyue [32]. However, in our study, we found that controlling for perceived social support did not appreciably attenuate the effect of chilla on depressive symptoms. While participation in chilla may be partially reflective of social support, we found that it was significantly associated with fewer depressive symptoms among women who were depressed during pregnancy regardless of their level of perceived social support. Thus, our results suggest that chilla captures more than perceived social support and may affect mental health through mechanisms other than social support.

It is likely that chilla affects mental health through multiple mechanisms, which are not fully captured by imagining chilla as a unidimensional proxy for social support. For example, mothers who are unable to participate in traditional postpartum practices may experience heightened role conflict, undermining their self-esteem [1]. Stern and Kruckman (1983) theorized that six primary elements influenced the presence or absence of PPD: a postpartum social support structure, recognition of vulnerability of the new mother, a mandated rest period, social seclusion, recognition of the role transition and social status of the new mother, and assistance with household tasks from female family, friends and midwives [1]. These specific cultural factors, they propose, buffer new mothers from PPD. In the Pakistani context, the postpartum practice of chilla encompasses these six primary protective elements, which suggests that we should consider the multiple dimensions of chilla and understand the full experience of chilla.

\section{The importance of postpartum practices}

Our findings emphasize the importance of understanding informal postpartum practices for mothers that are central to the postpartum period in Pakistan and other countries [10]. Given how common these practices are in Asia and around the world, there is a specific need to better understand the role of postpartum practices on maternal mental health and well-being during this transition period for mothers and how we can incorporate these practices into mental health interventions [3-5]. There is also a need to identify the characteristics that predict mothers' participation in these practices so that programs and interventions can focus on those less likely to participate. Supporting women's participation in chilla aligns with a global interest in using pre-existing structures to provide additional social support to women experiencing depression in the pre and postpartum period [34, 35]. Enhancing the practice of traditional postpartum practices can offer a sustainable and culturally-appropriate public health recommendation, potentially in both high and lower income settings.

\section{Strengths, limitations, and future work}

Our study has several strengths. First, it includes a longitudinal analysis from a population-representative sample. Second, we used standardized measures of depression symptom severity and MDE that have been validated in our target population. Third, it is the first, to date, to examine the effects of chilla participation, and one of the first to examine the effects of traditional postpartum practices, independently of social support on maternal mental health. Some limitations warrant discussion. First, as chilla and other postpartum practices are rarely quantitatively measured, it is not clear if our measurement of chilla captures the full experience. There is a need to more fully understand the quality and temporality of women's chilla experiences. Second, due to the lack of variability among chilla components, we were unable to decipher what components may be driving the association we see with maternal depression besides social support (i.e., diet or length of participation). Third, we were unable to assess if there is something unique about women who did not participate in chilla, which was only $11 \%$. Although our models control for social support and chilla components, there may be other elements of women's social environment that we are unable to capture. We do see that women with less education, lower SES, and higher depression severity are less likely to participate in chilla. Thus, what presumably prohibited women from participating in chilla that we were unable to measure may be driving the association between chilla participation and PPD. Fourth, it is possible that child sex can affect whether a mother participates in chilla, as mothers of female children are less likely to receive social support in the absence of chilla [36]. However, our sample size was too small to separately assess female and male births. Future research should consider the potential for effect measure modification by child sex. Lastly, we may have selection bias in our analytic sample, as women absent at three-month data collection may have been participating in chilla. However, given that chilla typically does not last more than 40 days, this is unlikely at the three-month follow-up.

\section{Conclusion}

Our study measures chilla's relationship with diagnosed MDE and depression symptom severity. We find that chilla was inversely associated with depression at 6 months postpartum above and beyond social support. Chilla was inversely associated with $\mathrm{MDE}$ among those not prenatally depressed and with lower symptom severity among those prenatally depressed. It is important for public health programs aimed at reducing PPD to consider the importance of the postpartum practices already in place in many regions of the world. Our findings signal an opportunity for maternal mental health interventions to draw upon these longstanding practices in creating culturally appropriate interventions for maternal mental health. 


\section{Appendix}

Table 4 Comparison of baseline characteristics between analytic and missing samples, Bachpan cohort, Pakistan

\begin{tabular}{|c|c|c|c|c|c|c|c|c|c|c|}
\hline & \multirow{2}{*}{\multicolumn{2}{|c|}{$\begin{array}{l}\text { Analytic Sample } \\
N=823\end{array}$}} & \multirow{2}{*}{\multicolumn{2}{|c|}{$\begin{array}{l}\text { Reappear at } 6 \text { Months } \\
N=105\end{array}$}} & \multicolumn{2}{|c|}{ Test of Difference+ } & \multirow{2}{*}{\multicolumn{2}{|c|}{$\frac{\text { Only in Baseline }}{N=226}$}} & \multicolumn{2}{|c|}{ Test of Difference++ } \\
\hline & & & & & \multirow[b]{2}{*}{ T-Test } & \multirow[b]{2}{*}{ Chi-Squared } & & & \multirow[b]{2}{*}{ T-Test } & \multirow[b]{2}{*}{ Chi-Squared } \\
\hline & N/Mean & $\% / S D$ & & & & & N/Mean & $\% / S D$ & & \\
\hline \multicolumn{11}{|l|}{ Demographic Characteristics } \\
\hline Maternal Age & 26.62 & 4.43 & 26.63 & 4.64 & -0.01 & & 27.05 & 4.91 & -1.25 & \\
\hline Maternal Education & & & & & & 4.29 & & & & \\
\hline None & 111 & 12.84 & 18 & 17.14 & & & 41 & 18.14 & & 3.47 \\
\hline Primary (1-5) & 159 & 18.33 & 23 & 21.9 & & & 44 & 19.47 & & \\
\hline Middle (6-8) & 157 & 18.67 & 19 & 18.1 & & & 39 & 17.26 & & \\
\hline Secondary $(9,10)$ & 209 & 25.66 & 28 & 26.67 & & & 56 & 24.78 & & \\
\hline Higher Secondary $(11,12)$ & 84 & 10.35 & 5 & 4.76 & & & 20 & 8.85 & & \\
\hline Tertiary (> 12) & 103 & 14.16 & 12 & 11.43 & & & 26 & 11.50 & & \\
\hline Parity & & & & & & 0.11 & & & & 3.06 \\
\hline First Pregnancy & 238 & 28.92 & 32 & 30.48 & & & 79.00 & 34.96 & & \\
\hline One or more children & 585 & 71.08 & 73 & 69.52 & & & 147.00 & 65.04 & & \\
\hline SES Quintiles & & & & & & 4.67 & & & & 4.07 \\
\hline Lowest & 153 & 16.93 & 27 & 25.71 & & & 50 & 22.12 & & \\
\hline Lower Middle & 163 & 18.08 & 17 & 16.19 & & & 51 & 22.57 & & \\
\hline Middle & 171 & 20.66 & 16 & 15.24 & & & 44 & 19.47 & & \\
\hline Upper Middle & 165 & 21.51 & 21 & 20 & & & 45 & 19.91 & & \\
\hline Highest & 171 & 22.82 & 24 & 22.86 & & & 36 & 15.93 & & \\
\hline Mother-in-law & & & & & & 0.33 & & & & $4.45^{*}$ \\
\hline Yes & 557 & 68.5 & 74 & 70.48 & & & 136 & 60.18 & & \\
\hline People in Room & 2.37 & 1.73 & 2.16 & 1.13 & 1.20 & & 2.72 & 2.56 & $-2.36^{*}$ & \\
\hline \multicolumn{11}{|l|}{ Depression Characteristics } \\
\hline SCID Baseline & 405 & 49.21 & 52 & 49.52 & & 0.00 & 105 & 46.88 & & 0.38 \\
\hline PHQ-9 Baseline & 8.73 & 6.68 & 8.22 & 6.79 & 0.74 & & 8.65 & 6.82 & 0.16 & \\
\hline
\end{tabular}

${ }^{*} p<.05,{ }^{* *}<.01,{ }^{* * *} p<.001 ;+$ Test of difference between analytic sample and those reappearing at 6 months; ++ Test of difference between analytic sample and those only at baseline; Abbreviations: Socioeconomic Status (SES), Patient Health Questionnaire (PHQ-9), Structured Clinical Interview for DSM-IV (SCID) 
Table 5 Comparison of chilla characteristics by baseline clinical depression, Bachpan cohort, Pakistan

\begin{tabular}{|c|c|c|c|c|c|c|}
\hline & \multicolumn{2}{|c|}{ Non-Depressed Baseline } & \multicolumn{2}{|c|}{ Depressed Baseline } & \multicolumn{2}{|c|}{ Test of Difference } \\
\hline & N/Mean & $\% / S D$ & N/Mean & $\% / S D$ & T-Test & Chi-Squared \\
\hline & $N=418$ & & $N=405$ & & & \\
\hline \multicolumn{7}{|l|}{ Chilla } \\
\hline \multirow[t]{2}{*}{ Yes } & 379 & 90.67 & 350 & 86.42 & & 3.67 \\
\hline & $N=379$ & & $N=350$ & & & \\
\hline Duration & & & & & $3.79^{* * *}$ & \\
\hline $1-7$ & 28 & 7.39 & 43 & 12.29 & & \\
\hline $8-14$ & 37 & 9.76 & 41 & 11.71 & & \\
\hline $15-20$ & 52 & 13.72 & 68 & 19.43 & & \\
\hline $21-25$ & 20 & 5.28 & 18 & 5.14 & & \\
\hline $26-30$ & 27 & 7.12 & 26 & 7.43 & & \\
\hline $31-35$ & 14 & 3.69 & 16 & 4.57 & & \\
\hline $36-40$ & 201 & 53.03 & 138 & 39.43 & & \\
\hline Chores & & & & & & 0.02 \\
\hline Yes & 323 & 85.22 & 297 & 84.86 & & \\
\hline Female Support & & & & & & 3.09 \\
\hline Yes & 358 & 94.46 & 328 & 93.71 & & \\
\hline Diet & & & & & & 0.18 \\
\hline Yes & 359 & 94.72 & 320 & 91.43 & & \\
\hline Satisfaction & & & & & & $16.89^{* * *}$ \\
\hline Yes & 334 & 88.13 & 268 & 76.57 & & \\
\hline
\end{tabular}


Table 6 Chilla participation and Postpartum Depression at Six Months, Bachpan cohort, Pakistan

\begin{tabular}{|c|c|c|c|c|c|c|c|c|c|c|c|c|}
\hline \multirow{3}{*}{ Panel A: SCID+ } & \multirow{2}{*}{\multicolumn{3}{|c|}{$\begin{array}{l}\text { Model A: Full Sample - Unadjusted } \\
N=729\end{array}$}} & \multirow{2}{*}{\multicolumn{3}{|c|}{$\begin{array}{l}\text { Model B: Full Sample - Adjusted } \\
N=729\end{array}$}} & \multirow{2}{*}{\multicolumn{3}{|c|}{$\begin{array}{l}\text { Model C: Non-depressed } \\
N=379\end{array}$}} & \multirow{2}{*}{\multicolumn{3}{|c|}{$\begin{array}{l}\text { Model D: Depressed } \\
N=350\end{array}$}} \\
\hline & & & & & & & & & & & & \\
\hline & OR & $\mathrm{Cl}$ & & OR & $\mathrm{Cl}$ & & OR & $\mathrm{Cl}$ & & $\mathrm{OR}$ & $\mathrm{Cl}$ & \\
\hline Chilla - Duration & $0.87^{* * *}$ & 0.80 & 0.94 & 0.92 & 0.84 & 1.01 & 0.87 & 0.75 & 1.01 & 0.95 & 0.85 & 1.06 \\
\hline Chilla - No Chores & 0.69 & 0.39 & 1.23 & 0.62 & 0.36 & 1.04 & 1.02 & 0.43 & 2.42 & $0.53^{*}$ & 0.28 & 0.99 \\
\hline Chilla - Female Help & 1.23 & 0.56 & 2.71 & & & & & & & & & \\
\hline Chilla - Diet & 0.72 & 0.32 & 1.62 & 0.93 & 0.44 & 1.98 & 1.09 & 0.29 & 4.07 & 0.72 & 0.31 & 1.67 \\
\hline Chilla Satifaction & 0.77 & 0.49 & 1.21 & 1.09 & 0.64 & 1.87 & 0.63 & 0.26 & 1.55 & 1.32 & 0.70 & 2.51 \\
\hline MSPSS & & & & $0.74^{* *}$ & 0.60 & 0.91 & $0.72^{*}$ & 0.53 & 0.97 & $0.77^{* *}$ & 0.63 & 0.95 \\
\hline SCID Baseline & & & & $2.42^{* * *}$ & 1.61 & 3.62 & 1.02 & 0.94 & 1.11 & 1.03 & 0.97 & 1.09 \\
\hline Maternal Age & & & & 1.04 & 0.99 & 1.09 & & & & & & \\
\hline Maternal Education & & & & $0.84^{*}$ & 0.73 & 0.97 & 0.88 & 0.69 & 1.11 & 0.93 & 0.77 & 1.12 \\
\hline Parity & & & & 1.12 & 0.61 & 2.06 & 1.29 & 0.61 & 2.71 & 1.27 & 0.66 & 2.44 \\
\hline SES & & & & 0.90 & 0.73 & 1.10 & 0.95 & 0.72 & 1.25 & 0.83 & 0.68 & 1.02 \\
\hline Mother-in-law & & & & 1.35 & 0.81 & 2.25 & 1.68 & 0.80 & 3.55 & 1.10 & 0.64 & 1.89 \\
\hline People in Room & & & & 1.02 & 0.93 & 1.11 & 1.12 & 0.98 & 1.28 & 0.93 & 0.79 & 1.08 \\
\hline Study Arm & & & & 0.92 & 0.61 & 1.39 & 0.91 & 0.48 & 1.73 & 0.87 & 0.55 & 1.40 \\
\hline Intercept & 0.98 & 0.38 & 2.49 & 0.82 & 0.09 & 7.70 & 0.92 & 0.05 & 17.88 & 3.08 & 0.35 & 27.02 \\
\hline Panel B: PHQ-9++ & Coefficient & $\mathrm{Cl}$ & & Coefficient & $\mathrm{Cl}$ & & Coefficient & $\mathrm{Cl}$ & & Coefficient & $\mathrm{Cl}$ & \\
\hline Chilla - Duration & -0.22 & -0.45 & 0.01 & -0.04 & -0.27 & 0.18 & -0.02 & -0.22 & 0.18 & -0.06 & -0.34 & 0.21 \\
\hline Chilla - No Chores & -0.56 & -2.29 & 1.18 & -0.59 & -2.05 & 0.87 & 0.47 & -0.69 & 1.64 & $-1.95^{*}$ & -3.57 & -0.34 \\
\hline Chilla - Female Help & 1.52 & -0.46 & 3.49 & & & & & & & & & \\
\hline Chilla - Diet & $-2.48^{* *}$ & -4.40 & -0.56 & $-1.49^{* *}$ & -2.63 & -0.34 & $-1.97^{*}$ & -3.75 & -0.19 & -0.71 & -2.88 & 1.46 \\
\hline Chilla Satifaction & -0.61 & -2.02 & 0.79 & 0.13 & -1.30 & 1.55 & -0.06 & -1.42 & 1.31 & 0.25 & -1.32 & 1.83 \\
\hline MSPSS & & & & $-0.69^{* * *}$ & -1.01 & -0.37 & $-0.54^{* *}$ & -0.97 & -0.12 & $-0.80^{* * *}$ & -1.31 & -0.29 \\
\hline PHQ-9 Baseline & & & & $0.24^{* * *}$ & 0.18 & 0.30 & $0.28^{* * *}$ & 0.19 & 0.38 & $0.20^{* * *}$ & 0.08 & 0.32 \\
\hline Maternal Age & & & & 0.05 & -0.03 & 0.13 & 0.05 & -0.05 & 0.15 & 0.06 & -0.08 & 0.20 \\
\hline Maternal Education & & & & -0.17 & -0.42 & 0.08 & -0.20 & -0.50 & 0.10 & -0.30 & -0.75 & 0.16 \\
\hline Parity & & & & 0.12 & -0.54 & 0.78 & 0.19 & -0.71 & 1.09 & 0.10 & -1.45 & 1.64 \\
\hline SES & & & & 0.03 & -0.33 & 0.38 & 0.12 & -0.24 & 0.47 & -0.06 & -0.55 & 0.44 \\
\hline Mother-in-law & & & & 0.35 & -0.32 & 1.01 & 0.32 & -0.61 & 1.24 & 0.38 & -0.95 & 1.71 \\
\hline People in Room & & & & 0.07 & -0.10 & 0.24 & -0.02 & -0.23 & 0.20 & 0.19 & -0.17 & 0.54 \\
\hline Study Arm & & & & $-0.78^{*}$ & -1.40 & -0.15 & $-0.91^{*}$ & -1.70 & -0.13 & -0.76 & -1.92 & 0.39 \\
\hline Intercept & 6.88 & 4.85 & 8.91 & 6.65 & 3.40 & 9.89 & 5.40 & 1.34 & 9.46 & 7.91 & 2.27 & 13.54 \\
\hline
\end{tabular}

${ }^{*} p<.05,{ }^{* *}<.01,{ }^{* * *} p<.001 ;$ Abbreviations: Multidimensional Scale of Perceived Social Support (MSPSS), Socioeconomic Status (SES), Patient Health Questionnaire $(P H Q-9)$, Structured Clinical Interview for DSM-IV (SCID)

\section{Abbreviations}

LMIC: Low- and middle-income countries; MDE: Major Depressive Episodes; MSPSS: Multidimensional Scale of Perceived Social Support; PHQ-9: Patient Health Questionnaire; PPD: Postpartum Depression; SCID: Structured Clinical Interview for the Diagnostic and Statistical Manual of Mental Disorders;

SES: Socioeconomic Status

\section{Acknowledgements}

The authors would like to thank the team at the Human Development Research Foundation (HDRF) including Rakshanda Liaqat, Tayyiba Abbasi, Maria Sharif, Samina Bilal, Quratul-Ain, Anum Nisar, Amina Bibi, Shaffaq Zufiqar, Sonia Khan, Ahmed Zaidi, Ikhlaq Ahmad, and Najia Atif for their meaningful contributions to the study's design and implementation. We also gratefully acknowledge the larger Bachpan and SHARE CHILD study teams.
Lastly, we are deeply grateful to the women, children, and communities that are a part of the Bachpan cohort.

\section{Authors' contributions}

$\mathrm{KL}, \mathrm{NA}, \mathrm{LZ}, \mathrm{AH}$, and $\mathrm{SB}$ conceptualized the analysis. $\mathrm{KL}$ designed and conducted the analysis with support from JAG and ELT. KL, NA, LZ, AH, EOC, SB, and JM drafted and revised the manuscript. SS and JM substantially contributed to the conception and design of the larger study and this analysis. All authors had access to all data and KL had final responsibility for the decision to submit for publication. All authors approved the final submission.

\section{Funding}

The larger study received funding from the National Institute of Mental Health [U19MH95687], and National Institute of Child Health and 
Development (NICHD) [R01 HD075875]. The study team is also supported by institutional grants awarded to the Carolina Population Center at the University of North Carolina at Chapel Hill from the NICHD (T32HD007168 and P2CHD050924). The funding bodies did not play any role in the design of the study, the collection of data, the analysis and interpretation of the data, or the writing of this manuscript.

\section{Availability of data and materials}

The datasets generated and/or analyzed during the current study are not publicly available due to ongoing data collection. However, data will be released upon completion of the study and, additionally, are available from the senior author Maselko on reasonable request.

\section{Ethics approval and consent to participate}

This study was approved by institutional review boards at the Human Development Research Foundation (Pakistan), the University of North Carolina at Chapel Hill (United States), and Duke University (United States). Written informed consent was required for study participation and was acknowledged with the participant's signature or, in the case of illiteracy, by a witness's signature.

\section{Consent for publication}

Not applicable.

\section{Competing interests}

The authors declare that they have no competing interests.

\section{Author details}

'Department of Epidemiology, Gillings School of Global Public Health, University of North Carolina at Chapel Hill, McGavran-Greenberg Hall, CB\# 7435, Chapel Hill, North Carolina NC 27599, USA. ${ }^{2}$ Carolina Population Center, University of North Carolina at Chapel Hill, 123 W. Franklin St, Chapel Hill, North Carolina NC 27516, USA. ${ }^{3}$ Department of Sociology, University of North Carolina at Chapel Hill, 102 Emerson Dr, Chapel Hill, North Carolina NC 27514, USA. ${ }^{4}$ School of Public Health, Yale University, 60 College St, New Haven, CT 06510, USA. ${ }^{5}$ Department of Biostatistics and Bioinformatics, Duke University, 2424 Erwin Road, Durham, North Carolina NC 27705, USA. ${ }^{6}$ Duke Global Health Institute, Duke University, Durham, North Carolina, USA. ${ }^{7}$ Department of Economics and Institute for Social and Economic Research, University of Essex, Wivenhoe Park, Colchester CO4 3SQ, UK. ${ }^{8}$ Human Development Research Foundation, H 06, Street 55, Sector F-7/4, Islamabad 44000, Pakistan. ${ }^{9}$ Health Services Academy, Islamabad, Pakistan.

\section{Received: 20 March 2019 Accepted: 8 January 2020} Published online: 15 January 2020

\section{References}

1. Stern G, Kruckman L. Multi-disciplinary perspectives on post-partum depression: an anthropological critique. Soc Sci Med. 1983;17:1027-41.

2. Mead M, Newton N. Cultural patterning of perinatal behavior. In: Richardson S, Guttmacher A, editors. Childbearing: Its Social and Psychological Aspects. Baltimore: Williams \& Wilkins; 1967. p. 142-244.

3. Niska K, Snyder M, Lia-Hoagberg B. Family ritual facilitates adaptation to parenthood. Public Health Nurs. 1998:15:329-37.

4. Bashiri N, Spielvogel AM. Postpartum depression: a cross-cultural perspective. Prim Care Update Ob Gyns. 1999;6:82-7.

5. Noble A, Rom M, Newsome-Wicks M, Engelhardt K, Woloski-Wruble A. Jewish Laws, customs, and practice in labor, delivery, and postpartum care. J Transcult Nurs. 2009;20:323-33.

6. Dennis CL, Fung K, Grigoriadis S, Robinson GE, Romans S, Ross L. Traditional postpartum practices and rituals: a qualitative systematic review. Women Health. 2007:3:487-502.

7. O'Hara MW, Wisner KL. Perinatal mental illness: definition, description and aetiology. Best Pract Res Clin Obstet Gynaecol. 2014;28:3-12.

8. Woody CA, Ferrari AJ, Siskind DJ, Whiteford HA, Harris MG. A systematic review and meta-regression of the prevalence and incidence of perinatal depression. J Affect Disord. 2017;219:86-92.

9. Fisher J, de Mello MC, Patel V, et al. Prevalence and determinants of common perinatal mental disorders in women in low-and lower-middle-income countries: a systematic review. Bull World Health Organ. 2012;90:139-49.
10. Grigoriadis S, Cindylee D, Kenneth F, et al. Postpartum cultural practices: a systematic review of the evidence. Ann General Psychiatry. 2008;7:S163.

11. Chien L-Y, Tai C-J, Ko Y-L, Huang C-H, Sheu S-J. Adherence to "Doing-themonth" practices is associated with fewer physical and depressive symptoms among postpartum women in Taiwan. Res Nurs Health. 2006;29:374-83.

12. Wong J, Fisher J. The role of traditional confinement practices in determining postpartum depression in women in Chinese cultures: a systematic review of the English language evidence. J Affect Disord. 2009; 116:161-9.

13. Yoshida K, Marks MN, Kibe N, Kumar R, Nakano H, Tashiro N. Postnatal depression in Japanese women who have given birth in England. J Affect Disord. 1997;43:69-77.

14. JRW F, Morrow MM, Nhu Ngoc NT, Hoang Anh LT. Prevalence, nature, severity and correlates of postpartum depressive symptoms in Vietnam. BJOG An Int J Obstet Gynaecol. 2004;111:1353-60.

15. Chee CYI, Lee DTS, Chong YS, Tan LK, Ng TP, Fones CSL. Confinement and other psychosocial factors in perinatal depression: a transcultural study in Singapore. J Affect Disord. 2005;89:157-66.

16. Uchino BN. Social support and health: a review of physiological processes potentially underlying links to disease outcomes. J Behav Med. 2006;29:377-87.

17. Qureshi R, Pacquiao D. Ethnographic study of experiences of Pakistani women immigrants with pregnancy, birthing, and postpartum Care in the United States and Pakistan. J Transcult Nurs. 2013;24:355-62.

18. Khadduri R, Marsh DR, Rasmussen B, Bari A, Nazir R, Darmstadt GL. Household knowledge and practices of newborn and maternal health in Haripur district, Pakistan. J Perinatol. 2008;28:182-7.

19. Parsons CE, Young KS, Rochat TJ. Postnatal depression and its effects on child development : a review of evidence from low- and middle-income countries. Br Med Bull. 2012;101:57-79.

20. Rahman A, lqbal Z, Harrington R. Life events, social support and depression in childbirth: perspectives from a rural community in the developing world. Psychol Med. 2003;33. https://doi.org/10.1017/\$0033291703008286.

21. Turner EL, Sikander S, Bangash O, et al. The effectiveness of the peerdelivered thinking healthy PLUS (THPP+) program for maternal depression and child socioemotional development in Pakistan: study protocol for a randomized controlled trial. Trials. 2016;17:1-11.

22. Spitzer RL, Williams JBW, Gibbon M, First MB. The structured clinical interview for DSM-III-R (SCID): I: history, rationale, and description. Arch Gen Psychiatry. 1992:49:624-9.

23. Rahman A, lqbal Z, Waheed W, Hussain N. Translation and cultural adaptation of health questionnaires. J Pak Med Assoc. 2008;53:142-7.

24. Gorman LL, O'Hara MW, Figueiredo B, et al. Adaptation of the structured clinical interview for DSM-IV disorders for assessing depression in women during pregnancy and post-partum across countries and cultures. $\mathrm{Br}$ 」 Psychiatry. 2004;184:s17-23.

25. Gallis JA, Maselko J, O'Donnell K, et al. Criterion-related validity and reliability of the Urdu version of the patient health questionnaire in a sample of community-based pregnant women in Pakistan. PeerJ. 2018;6:e5185.

26. Rahman A, labal Z, Bunn J, Lovel H, Harrington R. Impact of maternal depression on infant nutritional status and illness. Arch Gen Psychiatry. 2004;61:946.

27. Zimet G, Dahlem N, Zimet S, Farley G. The multidimensional scale of perceived social support. J Pers Assess. 1998;52:30-41.

28. Akhtar A, Rahman A, Husain M, Chaudhry IB, Duddu V, Husain N. Multidimensional scale of perceived social support: psychometric properties in a south Asian population. J Obstet Gynaecol Res. 2010;36:845-51.

29. Maselko J, Bates L, Bhalotra S, et al. Socioeconomic status indicators and common mental disorders: evidence from a study of prenatal depression in Pakistan. SSM Popul Heal. 2018:4:1-9.

30. Sikander S, Ahmad I, Bates LM, et al. Cohort Profile: Perinatal depression and child socioemotional development; the Bachpan cohort study from rural Pakistan. BMJ Open. 2019:9:e025644.

31. Rahman A, Malik A, Sikander S, Roberts C, Creed F. Cognitive behaviour therapy-based intervention by community health workers for mothers with depression and their infants in rural Pakistan: a cluster-randomised controlled trial. Lancet. 2008;372:902-9.

32. Lee T, Yip A, Leung T, Chung T. Ethnoepidemiology of postnatal depression. Br J Psychiatry. 2004;184:34-40.

33. Azidah AK, Shaiful BI, Rusli N, Jamil MY. Postnatal depression and sociocultural practices among postnatal mothers in Kota Bahru, Kelantan, Malaysia. Med J Malaysia. 2006;61:76-83. 
34. Chibanda D, Mesu P, Kajawu L, Cowan F, Araya R, Abas MA. Problem-solving therapy for depression and common mental disorders in Zimbabwe: piloting a task-shifting primary mental health care intervention in a population with a high prevalence of people living with HIV. BMC Public Health. 2011;11:1-10.

35. Martinez-schallmoser L. The Effect of Social Support and Acculturation on Postpartum Depression in Mexican American Women; 2003. https://doi.org/ 10.1177/1043659603257162.

36. Baranov V, Bhalotra S, Biroli P, Maselko J. Maternal Depression, Women's Empowerment, and Parental Investment: Evidence from a Large Randomized Control Trial, IZA Discussion Papers, No. 11187, Institute of Labor Economics (IZA), Bonn. 2017.

\section{Publisher's Note}

Springer Nature remains neutral with regard to jurisdictional claims in published maps and institutional affiliations.

Ready to submit your research? Choose BMC and benefit from:

- fast, convenient online submission

- thorough peer review by experienced researchers in your field

- rapid publication on acceptance

- support for research data, including large and complex data types

- gold Open Access which fosters wider collaboration and increased citations

- maximum visibility for your research: over $100 \mathrm{M}$ website views per year

At BMC, research is always in progress.

Learn more biomedcentral.com/submissions 